\title{
Hodgkin Lymphoma With Granulomas. Demystifying The Dilemma
}

\author{
Phiza Aggarwal $^{1 *}$, Mayank Sharma², Vinod Nimbran ${ }^{3}$ and Kamalpreet Kaur ${ }^{3}$ \\ ${ }^{1}$ Dept of Pathology, Govt Medical College \& hospital, Chandigarh(India) \\ ${ }^{2}$ Dept of Medicine, Indus International Hospital, Derabassi, Mohali, Punjab(India) \\ ${ }^{3}$ Dept of Radio-oncology, Indus International Hospital, Derabassi, Mohali, Punjab(India)
}

\begin{abstract}
Presence of granuloma in Hodgkin lymphoma is a common finding which is often a source of diagnostic confusion.There is no clearly defined strategy to approach this dual pathology. The present case describes a diagnostic approach using modalities like immunohistochemistry and Catridge based nucleic acid amplification test that helped reach to most likely cause of granuloma with underlying Hodgkin lymphoma.
\end{abstract}

Keywords: Hodgkin Lymphoma, Granulomatous Inflammation, Epithelioid Cell Granuloma, Malignancy

\section{Introduction}

Hodgkin lymphoma (HL) is a unique hematopoietic neoplasm arising from germinal center or post germinal center B cells. Histopathologically, the tumor is composed of characteristic multinucleate giant cells or large mononuclear cells in an inflammatory background referred to as Hodgkin and Reed-Sternberg (HRS) cells. ${ }^{[1]}$ However, atypical presentation of Hodgkin Lymphoma with formation of epithelioid cell granuloma along with tumor cells has been known in literature. ${ }^{[2-4]}$ Presence of granuloma often leads to delay in the accurate diagnosis and result in un-necessary treatments with antibiotics/ steroids. Even after widespread evidence of their association, there are no clear guidelines about the ideal diagnostic approach in this scenario. The present case describes another example of this diagnostic confusion in a patient who was diagnosed with Hodgkin lymphoma after comprehensive evaluation. The presenting discussion ought to demystify the mechanisms behind granuloma formation in malignancies and try to propose a diagnostic approach in a patient with granulomatous pathology on fine needle aspiration cytology of the lymph nodes.

\section{Case Report}

A 64 year female presented to medicine OPD with severe itching and red spots all over the body. There was no history of fever or loss of weight. On examination she had enlarged cervical, inguinal and axillary lymph nodes. Routine haematological tests showed a picture of pancytopenia with a hemoglobin of $7.0 \mathrm{gm} / \mathrm{dl}$, total leucocyte count of $3000 / \mathrm{cmm}$ and platelet count of $30,000 / \mathrm{cmm}$. X-ray chest was within normal limits but ultrasound abdomen showed gross hepatosplenomegaly with enlargement of retroperitoneal lymph nodes. The patient was non-reactive for HIV and serology for auto-immune disorders was negative. Fine needle aspiration cytology of the inguinal lymph node showed ill-defined non-caseating epithelioid cell granulomas without any atypical cells (figure 1). To confirm the diagnosis, lymph node biopsy was done which showed diffuse effacement of lymph node architecture with presence of characteristic binucleated RS and atypical mononuclear cells in the background of lymphocytes, plasma cells and eosinophils (Figure 2). The findings were suggestive of Hodgkin lymphoma. The tissue was also subjected to Catridge based nucleic acid amplification test (CBNAAT) which didn't show Mycobacterial tuberculosis DNA. Further, the blocks were subjected to immunohistochemistry (IHC) which showed CD15 \& CD30, OCT 2 and Pax 5 positivity which was diagnostic of Classic Hodgkin lymphoma - Mixed cellularity type. (Figure 3\&4) Bone marrow biopsy was done which also showed focal lymphoid infiltration along with eosinophils that was suggestive of Bone marrow invasion. The patients was planned for chemotherapy for Hodgkin Lymphoma but had a fatal cardiac arrest

\section{Discussion}

The present case describes an approach to diagnosis of Hodgkin lymphoma with co-occurrence of non-caseating epithelioid cell granulomas by comprehensive evaluation using histopathology, IHC and CBNAAT. Hodgkin lymphoma mostly affects the age group of 20-34 years with median age of diagnosis 39 years. ${ }^{[1]}$ Usually, it presents as low grade fever, weight loss and/or multiple 


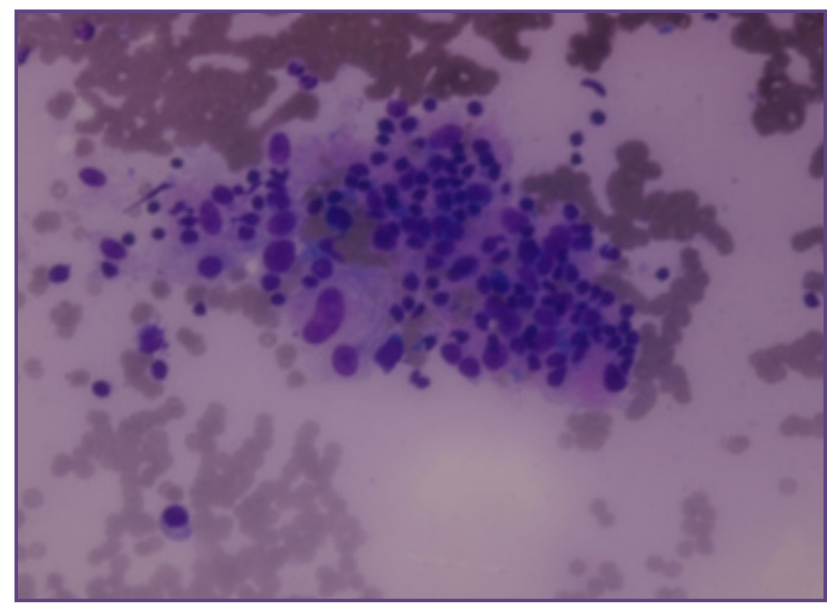

Fig. 1: Ill defined epithelioid cells along with lymphoid cells in FNA of inguinal lymph node (400 x).

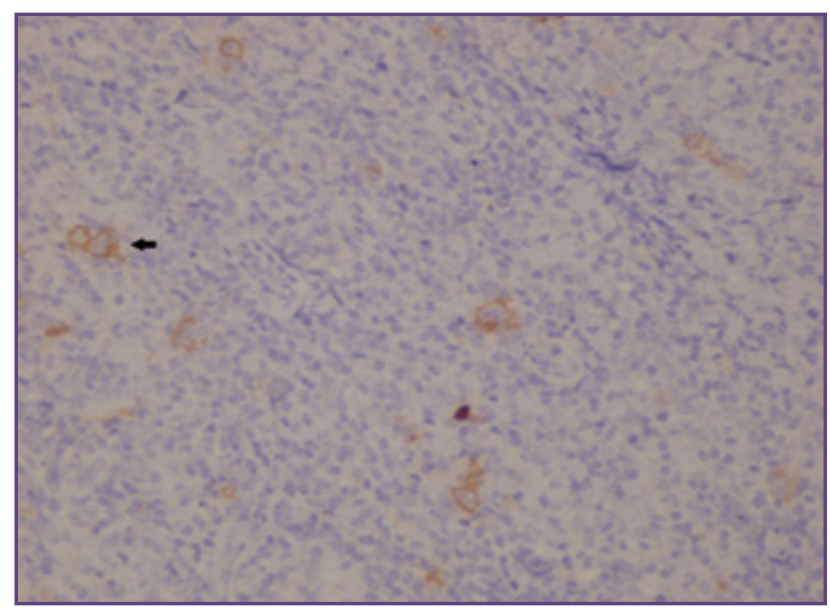

Fig. 3"CD15 positivity in Reed Sternberg Cells (100 x).

lymphadenopathy. Itching as a presenting symptom of lymphoma is rare phenomena as was seen in the present case. ${ }^{[5]}$ Presence of epithelioid cell granulomas in Hodgkin lymphoma is a well known event described in literature. [3, 4, 6] Besides Hodgkin Lymphoma, granulomatous inflammation have been known to complicate multiple malignancies like lung cancer $^{[7]}$, colonic carcinoma ${ }^{[8]}$ non-Hodgkin $\mathrm{T}$ cell lymphomas ${ }^{[9]}$ and breast cancer ${ }^{[10]}$. Epithelioid cell granuloma is histologically seen as a focal collection of inflammatory giant cells, macrophages, mononuclear cells and fibroblasts with or without caseous necrosis in the centre. These granulomas may form at the primary site of tumor or may develop at distant organs/ draining lymph nodes even without any evidence of malignancy ${ }^{[11,12]}$. Moreover, in Hodgkin lymphoma, granulomas may even sometimes precede the onset of the primary malignancy. ${ }^{[13]}$ Multiple possible causes/

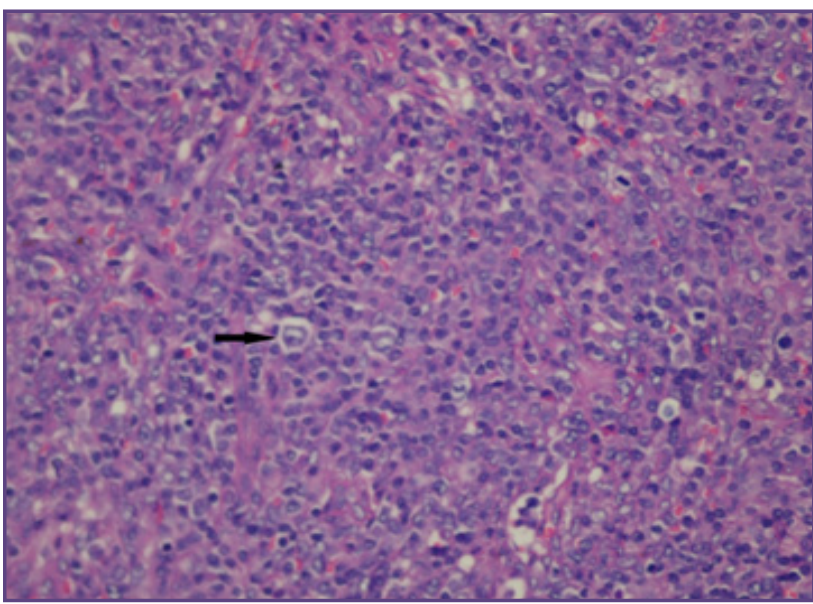

Fig. 2: H\&E stained inguinal lymph node biopsy shows atypical mononuclear cells ( arrow) in the polymorphous background of inflammatory cells $(100 \mathrm{x})$.

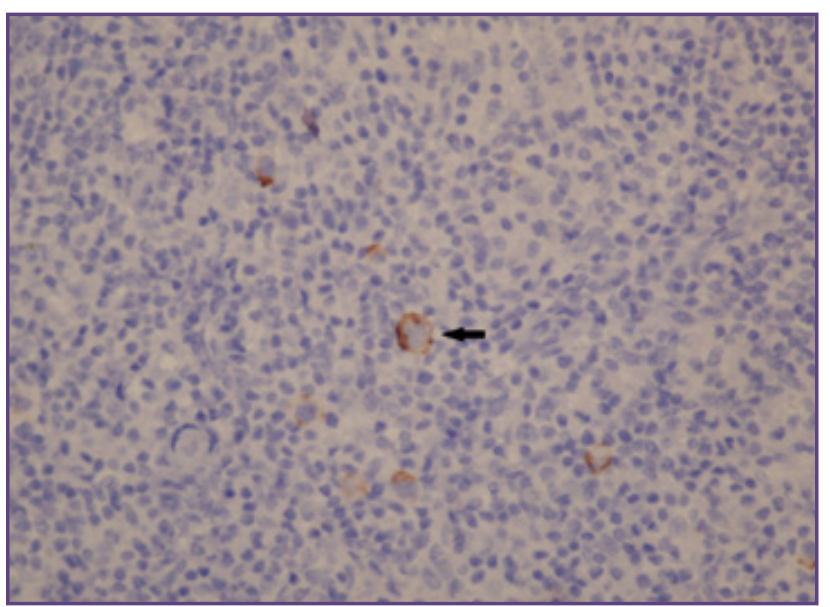

Fig. 4: Reed Sternberg cells (RS Cells) in Hodgkin lymphoma showing EBV positivity (100x).

mechanisms have been postulated for granuloma formation in underlying malignancy. Common causes include tumor related sarcoid reaction or sarcoid like lymphadenopathy, associated infections caused by bacteria (like tuberculosis), fungi and protozoa, foreign body reaction due to tumor necrosis and sarcoidosis. ${ }^{[12,14]}$ However, in majority of the cases no definite cause is identified leading to difficulty in treatment.

In spite of close association of granulomas with malignancy, initial detection of granulomas in patients without concurrent evidence of malignancy is usually dealt differently by different physicians with many ending further diagnostic workup and treating the patient with anti-tubercular treatment/steroids. Here, an important question arises, whether all patients with granulomas on FNAC should be subjected to biopsy and IHC as was done 
in the present patient? The ideal approach to diagnosis in such patients is not clearly defined in literature. It has been seen that the morphology of the granuloma does not differ between benign and malignant causes either on light or electron microscopy. However in most cases the granulomas are non-caseating in nature with many foreign body and Langhan's type giant cells. Their resemblance to sarcoid granulomas has prompted some authors to label them as sarcoid like granulomas or tumor related sarcoid reaction. ${ }^{[12]}$ In the present patient also, FNAC revealed non-caseating granulomas which prompted us to perform excision biopsy from the lymph node. Besides histopathology and IHC, the tissue was also subjected to Cartridge based nucleic acid amplification test (CBNAAT) that virtually excluded the diagnosis of tuberculosis. We could reasonably assign tumor related sarcoid reaction as the most probable cause of granulomas in the present case. Hence, it seems pertinent to perform histopathology in all patients with granulomas on FNAC but more consistently in those with clinical features suggestive of malignancy and/or presence of sarcoid like granuloma. However, IHC remains the gold standard for the confirmation in cases with equivocal presentation. Moreover, CBNAAT should be performed on all cytology/histopathology samples to rule out tuberculosis, especially in high TB burden countries like India. This evaluation becomes all the more important as tuberculosis, if confirmed, can be easily treated concurrently along with lymphoma. There is an urgent need to devise evidence based guidelines elaborating diagnostic strategy in patient with Granulomatous inflammation on needle aspiration of lymph nodes.

\section{References}

1. Shanbhag S, Ambinder RF. Hodgkin lymphoma: A review and update on recent progress. CA: 2017 Dec 1. doi: 10.3322/caac.21438. [Epub ahead of print]

2. Chopra R, Rana R, Zachariah A, Mahajan MK, Prabhakar BR. Epithelioid granulomas in Hodgkin's disease--prognostic significance. Indian J Pathol Microbiol 1995; 38: 427-33.
3. de Hemricourt E, De Boeck K, Hilte F, et al. Sarcoidosis and sarcoid-like reaction following Hodgkin's disease. Report of two cases. Mol Imaging Biol 2003; 5: 15-9.

4. Jensen MK, Pulczynski S, Johansen P. Suspected malignant lymphoma presenting with widespread granulomatous lesions in bones and lymph nodes and responding to combination chemotherapy. Acta haematologica 1996; 96: $237-41$.

5. Connors JM. Clinical manifestations and natural history of Hodgkin's lymphoma. Cancer J 2009; 15: 124-8.

6. Koo SE, Park CJ. Granulomatous bone marrow involvement of Hodgkin lymphoma, nodular sclerosis type. Blood Res 2017; 52: 82.

7. Dagaonkar RS, Choong CV, Asmat AB, et al. Significance of coexistent granulomatous inflammation and lung cancer. J Clin Pathol 2017; 70: 337-41.

8. Coyne JD. Colonic carcinoma with granulomatous (sarcoid) reaction. J Clin Pathol 2002; 55: 708-9.

9. Nyunt WW, Wong YP, Wan Jamaludin WF, Abdul Wahid SF. Diffuse large B cell lymphoma with chronic granulomatous inflammation. Malays J Pathol 2016; 38: 55-9.

10. Khurram M, Tariq M, Shahid P. Breast cancer with associated granulomatous axillary lymphadenitis: a diagnostic and clinical dilemma in regions with high prevalence of tuberculosis. Pathol Res Pract 2007; 203: 699-704.

11. Deen J, Banney L, Perry-Keene J. Palisading neutrophilic and granulomatous dermatitis as a presentation of Hodgkin lymphoma: A case and review. J Cutan Pathol 2017 Dec 11. doi: 10.1111/cup.13076. [Epub ahead of print]

12. Bhatia A, kumar Y, Kathpalia A. Granulomatous inflammation in lymph nodes draining cancer: A coincidence or a significant association. Int J Med and Medical Sci 2009; 1: $013-6$

13. Daly PA, O'Briain DS, Robinson I, Guckian M, Prichard JS. Hodgkin's disease with a granulomatous pulmonary presentation mimicking sarcoidosis. Thorax 1988; 43: 407-9.

14. Brincker $H$. Interpretation of granulomatous lesions in malignancy. Acta oncologica 1992; 31: 85-9

*Corresponding author:

Dr Phiza Aggarwal, Dept of Pathology, Block-E, Govt Medical College \& Hospital, Chandigarh(India) Pin: 160030

Phone: +919501004745

Email:drphiza@hotmail.com

Financial or other Competing Interests: None. 